\title{
Differences in HDL-cholesterol:apoA-I + apoA-II ratio and apoE phenotype with albuminuric status in Type I diabetic patients
}

\author{
S. S. Soedamah-Muthu' ${ }^{1}$ H. M. Colhoun ${ }^{1}$, M.-R. Taskinen ${ }^{2}$, B. Idzior-Walus ${ }^{3}$, J.H. Fuller ${ }^{1}$ and the EURODIAB \\ IDDM Complications Study Group
}

${ }^{1}$ EURODIAB, Royal Free and UCL medical school, London, UK

${ }^{2}$ University of Helsinki, Department of Medicine, Helsinki, Finland

${ }^{3}$ Jagiellonian University, Krakow, Poland

\section{Abstract}

Aims/hypothesis. To examine whether the HDL-cholesterol:apoA-I + apoA-II ratio and the $\varepsilon 2$ allele are related to albuminuria at baseline and whether they are risk factors for progression of albuminuria in a cohort study of patients with Type I (insulin-dependent) diabetes mellitus.

Methods. At baseline, the study cohort comprised 617 patients, aged 15-60 years, from seven European diabetic centres of the EURODIAB study. Albumin excretion rate, measured in a central laboratory, was categorised as normoalbuminuria at $20 \mu \mathrm{g} / \mathrm{min}$ or less, microalbuminuria between 20 and $200 \mu \mathrm{g} / \mathrm{min}$ or macroalbuminuria at $200 \mu \mathrm{g} / \mathrm{min}$ or over. Of the 250 patients who were normoalbuminuric at baseline and had follow-up albuminuria measurements, 34 patients were defined as early progressors.

Results. At baseline, the mean HDL-cholesterol:apoA-I + apoA-II ratio was lower in macroalbuminuric patients $(0.79,95 \%$ CI:0.74-0.83) compared with normoalbuminuric (0.88, $95 \%$ CI:0.87-0.90) patients $(p=0.0002$, adjusted for age and sex). At follow-up, 34 patients who progressed from normoalbuminuria to microalbuminuria or macroalbuminuria also had a slightly lower baseline ratio $(0.85,95 \%$ CI:0.80-0.89) than those 216 who remained normoalbuminuric $(0.89, \quad 95 \%$ CI:0.87-0.92) (adjusted $p=0.08$ ). Neither of these relations were independent of LDL-cholesterol or fasting triglyceride. There was no association of the $\varepsilon 2$ allele with albuminuria either at baseline $(\mathrm{OR}=1.4, \quad 95 \%$ CI:0.7-2.8) or with progression of albuminuria (OR $=0.4,95 \%$ CI:0.1-3.5).

Conclusion/interpretation. There is an inverse relation of HDL-cholesterol:apoA-I + apoA-II ratio with albuminuria at baseline. This lower ratio in microalbuminuric or macroalbuminuric patients could contribute to the increased risk of cardiovascular disease associated with nephropathy. There is weak evidence that HDL-composition is a risk factor for progression of albuminuria and no association of the $\varepsilon 2$ allele with diabetic nephropathy. [Diabetologia 2000 43: 1353-1359]

Keywords HDL-cholesterol, apolipoprotein E phenotype, $\varepsilon 2$ allele, apolipoproteins, albuminuria, Type I diabetes, apolipoprotein A-I, apolipoprotein A-II, diabetic nephropathy, progression.
Received: 24 April 2000 and in revised form: 6 July 2000

Corresponding author: S.S. Soedamah-Muthu, EURODIAB, Department of Epidemiology and Public-Health, Royal Free and University College London medical school, 1-19 Torrington Place, London WC1E 6BT, United Kingdom Abbreviations: apo, Apolipoprotein.
Diabetic nephropathy is associated with cardiovascular morbidity and mortality [1,2]. It is also associated with increased concentrations of serum total and LDL-cholesterol, triglyceride and apolipoprotein (apo)B $[3,4,5,6]$. In macroalbuminuria HDL-cholesterol is reduced but in microalbuminuric patients HDL-cholesterol has been reported to be reduced $[3,4]$, unchanged $[7,8]$ or increased [9] compared with normoalbuminuric patients [5]. 
An important question is whether there are also abnormalities of lipoprotein composition which contribute to the risk of diabetic nephropathy. Apolipoprotein A-I and apolipoprotein A-II make up over $85 \%$ of the protein found in HDL. The measurement of these apolipoproteins in plasma in conjunction with HDL-cholesterol provides a surrogate assessment of HDL composition [10]. In one previous study the HDL-cholesterol:apoA-I + apoA-II ratio was statistically significantly lower in those with microalbuminuria and macroalbuminuria compared with normoalbuminuric patients with Type I (insulin-dependent) diabetes mellitus [11]. We have examined whether albuminuria is associated with derangement of HDL-cholesterol:apoA-I + apoA-II ratio at baseline in a cohort of 617 Type I diabetic patients.

In addition to lipid abnormalities arising secondary to nephropathy there is also some evidence to suggest that lipid abnormalities are important in the initiation and progression of nephropathy [5]. Triglyceride-rich lipoproteins have been associated with diabetic nephropathy $[3,4,7,8]$. There are no previous studies examining whether HDL-cholesterol:apoA$\mathrm{I}+$ apoA-II ratio is prospectively associated with nephropathy. We therefore examined this question in 250 Type I diabetic patients of the original cohort of 617 patients who were normoalbuminuric at baseline and had follow-up albuminuria data available.

There is evidence that diabetic nephropathy is partly genetically determined [12]. Given the data suggesting a role for lipids in nephropathy progression, genes involved in lipoprotein metabolism are candidates for nephropathy. Apolipoprotein $\mathrm{E}$ is a component of triglyceride-rich lipoproteins, chylomicrons, very low-density lipoproteins and their remnants. It is also found to a lesser extent in high-density lipoproteins $[13,14]$. The gene for apoE, located on chromosome 19, has three common alleles, $\varepsilon 2$, $\varepsilon 3$, and $\varepsilon 4$, which code for three major isoforms, resulting in six common genotypes $[13,14]$. The presence of the $\varepsilon 2$ allele results in an accumulation of remnants of triglyceride-rich lipoprotein particles and a decrease in plasma LDL concentration [14]. Because the $\varepsilon 2$ allele is associated with an increased concentration of triglyceride $[15,16]$ it is a candidate gene for the development of nephropathy. Three small studies in Type I diabetic patients have found conflicting evidence on whether the $\varepsilon 2$ allele is a risk factor for nephropathy $[17,18,19]$. Therefore in this study we also examined whether the $\varepsilon 2$ allele is associated with albuminuria, cross-sectionally and prospectively.

\section{Subjects and methods}

Study design and subjects. Full details of the design and recruitment in the EURODIAB IDDM Complications Study have been published elsewhere [20]. This was a cross-sectional clin- ic-based study, carried out in 1989-1991. It was set up to explore risk factors for diabetic complications in 3250 Type I diabetic patients who were selected at random, aged 15 to 60 years and attending 31 clinics in 16 European countries. Sampling was stratified by age, sex and diabetes duration. At each clinic, informed consent from all patients and ethics committee approval for the study were obtained. Type I diabetes was defined as diabetes diagnosed before the age of 36 years with a continuous need for insulin 1 year after diagnosis.

Study cohort. Serum samples for the analysis of apolipoproteins, were available from seven of the EURODIAB centres $(n=617)$, which formed the baseline cohort in this study. The sub-sample $(n=617)$ did not differ from the total EURODIAB sample $(n=3250)$, in age, sex, duration of diabetes, albuminuria, $\mathrm{HbA}_{1 \mathrm{c}}$, systolic blood pressure or BMI.

Follow-up study. Approximately 6-8 years after the baseline investigations, the patients were traced and invited back for re-examination. Of the 617 patients, 426 patients were normoalbuminuric at baseline and of these 250 patients had followup albuminuria measurements, which allowed progression to be defined. Of the remaining 176 patients, 11 patients had died, 45 were ascertained to be alive but were not examined, and 120 were examined but follow-up albuminuria data were not available because of inadequate sample collection or concurrent infection. Progression from normoalbuminuria at baseline to microalbuminuria or macroalbuminuria was assessed by two 24-h urine collections (only one was done at baseline), measuring urinary albumin excretion after a mean follow-up period of 7.4 years. The term 'early progressors' was used for patients who progressed from normoalbuminuria to microalbuminuria or macroalbuminuria at follow-up. The term 'non-progressors' was used for patients who stayed normoalbuminuric at follow-up. Of the 250 patients who were normoalbuminuric at baseline and had follow-up albuminuria measurements, 34 were early progressors and 216 were nonprogressors. The baseline distribution of the $\varepsilon 2$ allele and the HDL composition was similar in those 250 patients compared with the total group $(n=617)$.

Measurements. Body Mass Index (BMI) was calculated (kg/ $\mathrm{m}^{2}$ ). Blood pressure was measured by a random zero sphygmomanometer (Hawskley, Lancing, UK) with the patient seated. Subjects conducted a single timed 24-h urine collection for the calculation of the albumin excretion rate (AER) after excluding proteinuria due to urinary tract infection. Urinary albumin was measured centrally by an immunoturbidimetric method [21] (Sanofi Diagnostics Pasteur, Minneapolis, Minn., USA). Albumin excretion rate was categorised as normoalbuminuria at $20 \mu \mathrm{g} / \mathrm{min}$ or less, microalbuminuria between 20 and $200 \mu \mathrm{g} / \mathrm{min}$ and macroalbuminuria at $200 \mu \mathrm{g} / \mathrm{min}$ or more. The $\mathrm{HbA}_{1 \mathrm{c}}$ value was measured by an enzyme immunoassay using a monoclonal antibody against $\mathrm{HbA}_{1 \mathrm{c}}$ [22]. A single venous blood sample was taken for the measurement of plasma lipids and $\mathrm{HbA}_{1 \mathrm{c}}$. Blood was collected in EDTA tubes after an overnight fast and before the morning insulin injection. Plasma was separated by centrifugation at $1500 \mathrm{~g}$ for $10 \mathrm{~min}$ at ambient temperature. Aliquots of whole blood, plasma and urine were stored at $-20^{\circ} \mathrm{C}$ at each centre until transported to the central laboratory on solid carbon dioxide. On receipt, samples were kept at $-20^{\circ} \mathrm{C}$ until analysed [23]. The triglyceride [24] and cholesterol [25] concentration of plasma and the cholesterol concentration of HDL [26] were assayed by standard enzymatic methods (Boehringer Mannheim, Lewes, UK) using a cobas-bio centrifugal analyser (Roche, Welwyn Garden City, Herts, UK). For HDL, samples with triglyceride 
Table 1. Baseline characteristics of normoalbuminuric and albuminuric patients at baseline

\begin{tabular}{|c|c|c|c|c|}
\hline & Normo & Micro & Macro & $p$ value \\
\hline Number of subjects & 426 & 124 & 52 & \\
\hline $\operatorname{Sex}(\% \operatorname{men})^{\mathrm{a}}$ & 49 & 58 & 52 & 0.2 \\
\hline Age (years) & $33 \pm 10$ & $32 \pm 10$ & $35 \pm 10$ & 0.2 \\
\hline $\mathrm{BMI}\left(\mathrm{kg} / \mathrm{m}^{2}\right)$ & $24 \pm 3$ & $25 \pm 4$ & $25 \pm 3$ & $0.03^{\mathrm{c}}$ \\
\hline $\mathrm{HbA}_{1 \mathrm{c}}(\%)$ & $6 \pm 2$ & $7 \pm 2$ & $7 \pm 2$ & $0.0001^{\mathrm{c}}$ \\
\hline Systolic blood pressure $(\mathrm{mmHg})$ & $119 \pm 16$ & $128 \pm 19$ & $134 \pm 18$ & $0.0001^{\mathrm{c}}$ \\
\hline $\mathrm{c}(\mathrm{mmol} / \mathrm{l})$ & $5.2 \pm 1.0$ & $5.4 \pm 1.2$ & $6.3 \pm 1.5$ & $0.0001^{\mathrm{c}}$ \\
\hline LDL-c (mmol/l) & $3.3 \pm 0.9$ & $3.3 \pm 0.9$ & $4.5 \pm 1.3$ & $0.0001^{\mathrm{c}}$ \\
\hline HDL-c (mmol/l) & $1.5 \pm 0.4$ & $1.5 \pm 0.5$ & $1.4 \pm 0.4$ & 0.2 \\
\hline Fasting triglyceride ${ }^{\mathrm{b}}(\mathrm{mmol} / \mathrm{l})$ & $0.9 \pm 1.6$ & $1.1 \pm 1.7$ & $1.6 \pm 1.6$ & $0.0001^{\mathrm{c}}$ \\
\hline apoA-I (mmol/l) & $1.4 \pm 0.3$ & $1.4 \pm 0.3$ & $1.4 \pm 0.3$ & 0.4 \\
\hline apoA-II (mmol/l) & $0.3 \pm 0.1$ & $0.4 \pm 0.1$ & $0.4 \pm 0.1$ & $0.02^{\mathrm{c}}$ \\
\hline
\end{tabular}

${ }^{\mathrm{a}}$ Chi-squared test, ${ }^{\mathrm{b}}$ Geometric mean, all values are means $\pm \mathrm{SD},{ }^{\mathrm{c}} p$ value $<0.05$ is significant with ANOVA. $\mathrm{c}=$ cholesterol

concentrations more than $3 \mathrm{mmol} / \mathrm{l}$ were diluted with $0.15 \mathrm{~mol} / \mathrm{l}$ sodium chloride solution before chemical precipitation. Lowdensity lipoprotein cholesterol (LDL-cholesterol) was calculated from Friedewald's formula [27]. Stored serum samples were analysed for apolipoproteins (A-I and A-II) by an immunoturbidimetric method $(\mathrm{mmol} / \mathrm{l})$ with commercially available kits (Boehringer Mannheim) [28, 29]. The ratio of HDL-cholesterol and the sum of apoA-I and apoA-II was calculated (HDL-cholesterol:apoA-I + apoA-II).

Apolipoprotein E phenotype was measured using a rapid micromethod. The method is based on isoelectric focusing of either delipidated or guanide-HCL-treated serum in a horizontal slab gel system, followed by immunoblotting using either polyclonal or monoclonal anti-apolipoprotein $\mathrm{E}$ antibodies as the first antibody [30]. Apolipoprotein E genotype was not measured directly but derived from the measurement of apoE phenotype. The laboratory measurements were conducted in a central laboratory in Helsinki, Finland.

Statistical analyses. All analyses were done using the SAS statistical package (SAS, Cary, N.C., USA). Log transformations were used for variables with skewed distributions. Apolipoprotein $\mathrm{E}$ phenotypes were expressed as E3/1, E3/2, E3/3, E4/2, $\mathrm{E} 4 / 3, \mathrm{E} 4 / 4$. For the patients with $\varepsilon 2$ allele the E3/2 and E4/2 phenotypes were combined as one group [31]. The chi-squared test for trend was used to analyse the differences between ApoE phenotype and albuminuria. Logistic regression analysis was used for further analysis of differences between the $\varepsilon 2$ allele and albuminuria. In the final logistic model adjustment was made for age, sex, LDL-cholesterol, fasting triglyceride and HDL-cholesterol.

Analysis of covariance (ANCOVA) was used to test for associations between HDL-cholesterol:apoA-I + apoA-II ratio and albuminuria at baseline and with progression of albuminuria. These analyses were computed by fitting linear regression models for the HDL-cholesterol:ApoA-I + ApoA-II ratio and including indicator variables for the albuminuria groups. The following covariates were entered separately into the model: AER (log transformed), age, sex, duration of diabetes, $\mathrm{HbA}_{1 \mathrm{c}}$, BMI, systolic blood pressure, LDL-cholesterol, fasting triglyceride and smoking. A $p$ value of less than 0.05 was considered statistically significant.

\section{Results}

Distribution of characteristics between normoalbuminuric and albuminuric patients at baseline. There was a significant difference between patients with normoalbuminuria $(n=426)$, microalbuminuria $(n=$ $124)$ and macroalbuminuria $(n=52)$ for the following variables: duration of diabetes, $\mathrm{BMI}, \mathrm{HbA}_{1 \mathrm{c}}$, systolic blood pressure, diastolic blood pressure, smoking, total cholesterol, LDL-cholesterol, fasting triglyceride and apoA-II. The LDL-cholesterol, fasting triglyceride and apoA-II concentrations were increased in microalbuminuric or macroalbuminuric patients compared with normoalbuminuric patients, whereas apoA-I was similar in all albuminuria groups (Table 1).

Distribution of characteristics between early progressors and non-progressors. Duration of diabetes $(p=0.04)$ and $\mathrm{HbA}_{1 \mathrm{c}}$ at baseline $(p=0.0008)$ were the only two variables that showed a significant difference between early progressors $(n=34)$ and nonprogressors $(n=216)$ (data not shown).

HDL-cholesterol:apoA-I + apoA-II ratio and albuminuria at baseline. The HDL-cholesterol:apoAI + apoA-II ratio was inversely related to albuminuria at baseline, with mean values of 0.88 (95\% CI: $0.87-0.90), 0.87$ (95\% CI: 0.84-0.89) and 0.79 (95\% CI: $0.74-0.83)$ in those with normoalbuminuria, microalbuminuria and macroalbuminuria, respectively $(p=0.0002$, adjusted for age and sex $)$ (Fig. 1). This relation was mainly due to the lower HDL-cholesterol:apoA-I + apoA-II ratio in the macroalbuminuria group compared with the microalbuminuria and normoalbuminuria groups ( $p=0.03$ and $p=0.0001$, respectively). Potential confounding variables, duration of diabetes, $\mathrm{HbA}_{1 \mathrm{c}}$, BMI, systolic blood pressure, LDL-cholesterol, fasting triglyceride and smoking, were entered in the model one by one to analyse 


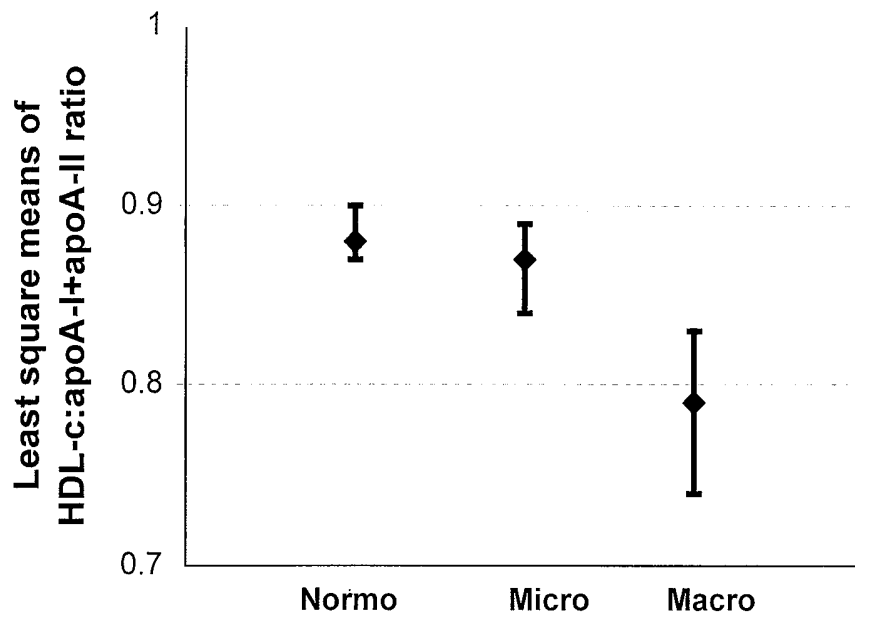

Fig.1. HDL-cholesterol:apoA-I + apoA-II ratio in relation to albuminuria at baseline, adjusted for age and $\operatorname{sex}(p=0.0002)$

Table 2. Frequency distribution of different apoE phenotypes in study cohort $(n=617)$

\begin{tabular}{lrcr}
\hline ApoE phenotype & Men & Women & Total \\
\hline E3/E1 & $1 \%$ & $0 \%$ & $1 \%$ \\
E3/E2 & $13 \%$ & $14 \%$ & $13 \%$ \\
E3/E3 & $62 \%$ & $54 \%$ & $58 \%$ \\
E4/E2 & $2 \%$ & $1 \%$ & $2 \%$ \\
E4/E3 & $20 \%$ & $28 \%$ & $24 \%$ \\
E4/E4 & $2 \%$ & $2 \%$ & $2 \%$ \\
\hline
\end{tabular}

which of the variables attenuated the relation between HDL-cholesterol:apoA-I + apoA-II and albuminuria at baseline (adjusted for age and sex). Lowdensity lipoprotein cholesterol or fasting triglyceride were the only variables that attenuated this relation. The HDL-cholesterol:apoA-I + apoA-II ratio was not inversely related to albuminuria after adjustment for LDL-cholesterol, with mean values of 0.86 (95\% CI: 0.84-0.88), 0.83 (95\% CI: 0.80-0.86), 0.84 (95\% CI: 0.78-0.89) in those with normoalbuminuria, microalbuminuria and macroalbuminuria, respectively $(p=0.2)$. After adjustment for fasting triglyceride, the mean values of the HDL-cholesterol:apoAI + apoA-II ratio were similar for normoalbuminuria, microalbuminuria and macroalbuminuria $(p=0.97)$.

HDL-cholesterol:apoA-I + apoA-II ratio and progression of albuminuria. Early progressors showed a slightly lower HDL-cholesterol:apoA-I + apoA-II ratio $(0.85,95 \%$ CI: $0.80-0.89)$ at baseline than nonprogressors $(0.89,95 \%$ CI: $0.87-0.92)(p=0.08$, adjusted for AER, age and sex). On adjustment for LDL-cholesterol or fasting triglyceride this difference was abolished. The mean LDL-cholesterol concentration at baseline was $3.8 \mathrm{mmol} / \mathrm{l}(1.0 \mathrm{SD})$ and the mean triglyceride concentration was $1.0 \mathrm{mmol} / \mathrm{l}$ (1.8 SD). There was no statistically significant difference in baseline LDL-cholesterol or fasting triglyceride between early progressors and non-progressors, the lipids were, however, highly correlated. The correlation coefficients between HDL-cholesterol:apoA-I + apoA-II ratio and triglyceride -0.6 $(p=0.0001)$ and with LDL-cholesterol -0.4 $(p=0.0001)$.

Apolipoprotein $\varepsilon 2$ allele. The frequency distribution of apoE phenotypes is given in Table 2. The frequency of patients with at least one $\varepsilon 2$ allele $(\mathrm{E} 2 / 3+\mathrm{E} 4 /$ $2)$ was $15 \%(n=92)$. Patients with the $\varepsilon 2$ allele had a significantly lower systolic blood pressure $(p=0.04)$, higher smoking rates $(p=0.03)$ and lower LDL-cholesterol concentrations $(p=0.0006)$ than those without the $\varepsilon 2$ allele. Even after adjustment for age and sex, the LDL-cholesterol concentration was significantly lower in patients with an $\varepsilon 2$ allele than in patients without $(p=0.0003)$ (data not shown).

Albuminuria was present in $34 \%$ of the patients with an $\varepsilon 2$ allele $(n=92)$ compared with $29 \%$ of the patients without an $\varepsilon 2$ allele $(p$ for trend $=0.4)$. In a logistic regression model (Table 3 ) there was no association between $\varepsilon 2$ allele and either albuminuria at baseline or progression of albuminuria, with or without adjustment for lipids. Further adjustment for systolic blood pressure did not alter this relation (data not shown).

\section{Discussion}

The main findings in this study are that patients with albuminuria have a significantly lower HDL-cholesterol:apoA-I + apoA-II ratio at baseline. This altered composition could contribute to the increased risk of coronary artery disease in these patients. The relation between HDL-cholesterol:apoA-I + apoA-II ratio

Table 3. The association of the $\varepsilon 2$ allele with albuminuria

\begin{tabular}{|c|c|c|c|}
\hline & & $\begin{array}{l}\text { OR }(95 \% \mathrm{CI}) \\
\text { baseline }\end{array}$ & $\begin{array}{l}\text { OR }(95 \% \mathrm{CI}) \\
\text { progression }\end{array}$ \\
\hline Odds ratio for albuminuria $(\mathrm{AER}>20 \mu \mathrm{g} / \mathrm{min})$ associated with ha & any $\varepsilon 2$ allele & $1.3(0.8-2.0)$ & $0.5(0.1-1.7)$ \\
\hline As above adjusted for age, sex, LDL-c, fasting triglyceride ${ }^{\mathrm{a}}$, HDL & & $1.4(0.7-2.8)$ & $0.4(0.1-3.5)$ \\
\hline
\end{tabular}


and albuminuria is not independent of LDL-cholesterol or fasting triglyceride. There is only a weak non-statistically significant association between HDL-cholesterol:apoA-I + apoA-II ratio and progression of albuminuria and no association between the $\varepsilon 2$ allele and albuminuria.

There are only two cross-sectional studies which have analysed the HDL-cholesterol:apoA-I + apoAII ratio in patients with Type I diabetes $[11,32]$. In one of these studies, the HDL-cholesterol:apoA$\mathrm{I}+$ apoA-II ratio was used as a measurement of the composition of HDL but this ratio was not analysed in relation to nephropathy [32]. In the other study, a significantly lower HDL-cholesterol:apoA-I + apoAII ratio was found in microalbuminuric or macroalbuminuric patients compared with normoalbuminuric patients [11]. Whether this relation was independent of LDL-cholesterol or fasting triglyceride was not examined. After adjustment for LDL-cholesterol or fasting triglyceride in our study there was no longer a statistically significant relation between HDL-cholesterol:apoA-I + apoA-II ratio and albuminuria at baseline. These data are consistent with increasing triglyceride associated with nephropathy being the main determinant of the altered HDL composition possibly resulting from an accelerated exchange of cholesterol for triglyceride from HDL-cholesterol to triglyceride-rich lipoproteins $[33,34,35]$. We did not find any clear evidence that HDL compositional abnormalities are a risk factor for the initiation and progression of albuminuria although there was a weak non-statistically significant association. The number of early progressors was, however, very small, thereby reducing the power of our analysis $(n=34)$.

Studies of the absolute concentrations of apoA-I and apoA-II in patients with Type I diabetes and microalbuminuria have been inconsistent, finding reduced $[8,36]$, unchanged $[4,6,11]$ and increased $[37$, 38 ] concentrations of apolipoproteins in microalbuminuric patients compared with normoalbuminuric patients. In our larger study, we found similar apoAI concentrations and increased apoA-II concentrations in microalbuminuric patients compared with normoalbuminuric patients.

The absence of an association between the $\varepsilon 2$ allele and nephropathy found here is consistent with a previous study of 146 Type I diabetic patients [18]. Two previous studies $(n=162$ and 252) did, however, find an association [17, 19]. Two out of three studies in patients with Type II (non-insulin-dependent) diabetes mellitus found that the $\varepsilon 2$ allele was associated with increased risk of nephropathy [31, 39, 40]. One reason for these conflicting results is that previous studies had smaller numbers of diabetic patients with an $\varepsilon 2$ allele than our study $[17,18,19,31,39$, 40]. Another possibility is that the relation between the $\varepsilon 2$ allele and nephropathy is mediated through triglyceride. In patient groups where the $\varepsilon 2$ allele is a less important determinant of triglyceride concentrations, the association between the $\varepsilon 2$ allele and nephropathy could be weaker. We did not find a relation between the $\varepsilon 2$ allele and fasting triglyceride in our patients. The other three studies of $\varepsilon 2$ allele and nephropathy in Type I diabetes did not examine the relation between the $\varepsilon 2$ allele and triglyceride concentration $[17,18,19]$. Most previous studies in Type II diabetes, that found that the $\varepsilon 2$ allele is associated with the development of diabetic nephropathy also found an increased concentration of triglyceride in patients with any $\varepsilon 2$ allele compared with those without $[31,39]$ but they did not adjust for triglyceride.

We conclude that there is no relation between the $\varepsilon 2$ allele and albuminuria. Our data emphasise the necessity of showing the replicability of results in genotype-phenotype association studies.

Patients with albuminuria, along with increased triglyceride and LDL-cholesterol concentrations have compositional abnormalities in HDL emphasising the need for vigilant lipid lowering therapy in this patient group. The altered HDL composition associated with albuminuria could be important in the increased risk of cardiovascular disease in these patients.

Acknowledgements. We would like to thank all the centres and staff involved in the EURODIAB IDDM Complications Study. We would also like to thank the Wellcome Trust and European Union for their grant support and all staff and patients who took part in the study. M. Shipley is acknowledged for his statistical advice.

Centres and staff involved in this sub-study. P. Ebeling, M. Kylliäinen, T. Kyostio-Renvall, University Hospital of Helsinki, Finland. B. Idzior-Walus, B. Solnica, J. Sieradzki, K. Cyganek, Department of Metabolic Diseases, Jagiellonian University, Krakow, Poland. H.H.P.J. Lemkes, C. Roest, University Hospital of Leiden, The Netherlands. R. Navalesi, G. Penno, S. Bandinelli, R. Miccoli, Department of Endocrinology and Metabolism, Pisa, Italy. J. D. Ward, S. Tesfaye, S. Eaton, C. Mody, Royal Hallamshire Hospital, Sheffield, UK. J.H. Fuller, J. Holloway, L. Asbury, D. J. Betteridge, University College London, UK. S. Walford, E. V. Wardle, J. Sinclair, S. Hughes, New Cross Hospital, Wolverhampton, UK.

Steering committee members. J.H. Fuller (London), B. Karamanos, Chairman (Athens), A.-K. Sjolie (Aarhus), N. Chaturvedi (London), M. Toeller (Dusseldorf), G. Pozza Co-chairman (Milan), B. Ferriss (Cork), M. Porta (Turin), R. Rottiers (Gent), G. Michel (Luxembourg).

Co-ordinating centre. J.H. Fuller, N. Chaturvedi, J. Holloway, D. Webb, L. Asbury, University College London. 
Central laboratories. G.-C. Viberti, R. Swaminathan, P. Lumb, A. Collins, S. Sankaralingham, Guy's and St Thomas Hospital, London, UK.

\section{References}

1. Borch-Johnsen K, Kreiner S (1987) Proteinuria value as predictor of cardiovascular mortality in insulin-dependent diabetes. BMJ 294: 1651-1654

2. Jensen T, Borch-Johnsen K, Kofoed-Enevoldsen A, Deckert T (1987) Coronary heart disease in young Type I (insulin-dependent) diabetic patients with and without diabetic nephropathy: incidence and risk factors. Diabetologia 30: 144-148

3. Jensen T, Stender S, Deckert T (1988) Abnormalities in plasmas concentrations of lipoproteins and fibrinogen in Type I (insulin-dependent) diabetic patients with increased urinary albumin excretion. Diabetologia 31: 142-145

4. Watts GF, Naumova R, Slavin BM et al. (1989) Serum lipids and lipoproteins in insulin-dependent diabetic patients with persistent microalbuminuria. Diabet Med 6: 25-30

5. Groop PH (1998) Lipidaemia and diabetic renal disease. In: Mogensen CE (ed) The kidney and hypertension in diabetes mellitus. 4th edn. Kluwer Academic Publishers, Boston, pp 357-369

6. Gall MA, Rossing P, Hommel E et al. (1992) Apolipoprotein (a) in insulin-dependent diabetic patients with and without diabetic nephropathy. Scand J Clin Lab Invest 52: 513-521

7. Dullaart RP, Groener JE, Dikkeschei LD, Erkelens DW, Doorenbos H (1989) Increased cholesterylester transfer activity in complicated Type I (insulin-dependent) diabetes mellitus - its relationship with serum lipids. Diabetologia 32: $14-19$

8. Jones SL, Close CF, Mattock MB, Jarrett RJ, Keen H, Viberti GC (1989) Plasma lipid and coagulation factor concentrations in insulin-dependent diabetics with microalbuminuria. BMJ 298: 487-490

9. Jay RH, Jones SL, Hill CE et al. (1991) Blood rheology and cardiovascular risk factors in type I diabetes: relationship with microalbuminuria. Diabet Med 8: 662-667

10. Eckel RH, Albers JJ, Cheung MC, Wahl PW, Lindgren FT, Bierman EL (1981) High density lipoprotein composition in insulin-dependent diabetes mellitus. Diabetes 30: 132-138

11. Kahri J, Groop PH, Elliott T, Viberti G, Taskinen M-R (1994) Plasma cholesteryl ester transfer protein and its relationship to plasma lipoproteins and apolipoproteins and apolipoprotein A-I containing lipoproteins in IDDM patients with microalbuminuria and clinical nephropathy. Diabetes Care 17: 412-419

12. Chowdhury TA, Dyer PH, Kumar S, Barnett AH, Bain SC (1999) Genetic determinants of diabetic nephropathy. Clin Sci (Colch) 96: 221-230

13. Salo MK, Rantanen R, Huupponen T, Lehtimäki T, Jokela H (1993) Apolipoprotein E phenotypes and plasma lipids in diabetic children and adolescents. Eur J Pediatr 152: 564-568

14. Davignon J, Gregg RE, Sing CF (1988) Apolipoprotein E polymorphism and atherosclerosis. Arteriosclerosis 8: $1-21$

15. Assmann G, Schmitz G, Menzel HJ, Schulte H (1984) Apolipoprotein E polymorphism and hyperlipidemia. Clin Chem 30: 641-643
16. Eto M, Watanabe K, Iwashima Y et al. (1986) Apolipoprotein E polymorphism and hyperlipemia in type II diabetics. Diabetes 35: 1374-1382

17. Werle E, Fiehn W, Hasslacher C (1998) Apolipoprotein E polymorphism and renal function in German type 1 and type 2 diabetic patients. Diabetes Care 21: 994-998

18. Onuma T, Laffel LM, Angelico MC, Krolewski AS (1996) Apolipoprotein E genotypes and risk of diabetic nephropathy. J Am Soc Nephrol 7: 1075-1078

19. Chowdhury TA, Dyer PH, Kumar S et al. (1998) Association of apolipoprotein $\varepsilon 2$ allele with diabetic nephropathy in Caucasian subjects with IDDM. Diabetes 47: 278-280

20. EURODIAB IDDM Complications Study Group (1994) Microvascular and acute complications in IDDM patients: the EURODIAB IDDM Complications Study. Diabetologia 37: 278-285

21. Kearney EM, Mount JN, Watts GF, Slavin BM, Kind PR (1998) Simple immunoturbidimetric method for determining urinary albumin at low concentrations using Cobas-bio centrifugal analyser. J Clin Path 40: 465-468

22. John GW, Gray MR, Bates DL, Beacham JL (1993) Enzyme immunoassay: a new technique for estimating $\mathrm{HbA}_{1 \mathrm{c}}$. Clin Chem 39: 663-666

23. Koivisto VA, Stevens LK, Mattock M et al. (1996) Cardiovascular disease and its risk factors in IDDM in Europe. Diabetes Care 19: 689-697

24. Buculo G, David H (1973) Quantitative determination of serum triglycerides by the use of enzymes. Clin Chem 19: 476-482

25. Siedel J, Hägele EO, Ziegenhorn J, Wahlefeld AW (1983) Reagent for the enzymatic determination of serum total cholesterol with improved lipolytic efficiency. Clin Chem 29: 1075-1080

26. Warnick GR, Albers JJ (1978) A comprehensive evaluation of the heparin-manganese precipitation procedure for estimating high density lipoprotein cholesterol. J Lipid Res 19: 65-76

27. Friedewald WT, Levy RI, Fredrickson DS (1972) Estimation of the concentration of low-density lipoprotein cholesterol in plasma, without use of the preparative ultracentrifuge. Clin Chem 18: 499-502

28. Syvänne M, Kahri J, Virtanen KS, Taskinen M-R (1995) HDLs containing apolipoproteins A-I and A-II (LpA-I:AII) as markers of coronary artery disease in men with noninsulin dependent diabetes mellitus. Circulation 92: 364-370

29. Lahdenperä S, Groop PH, Tilly-Kiesi M et al. (1994) LDL subclasses in IDDM patients: relation to diabetic nephropathy. Diabetologia 37: 681-688

30. Havekes LM, de Knijff P, Beisiegel U, Havinga J, Smit M, Klasen E (1987) A rapid micromethod for apolipoprotein $\mathrm{E}$ phenotyping directly in serum. J Lipid Res 28: 455-463

31. Eto M, Horita K, Morikawa A et al. (1995) Increased frequency of apolipoprotein $\varepsilon 2$ allele in non-insulin dependent diabetic (NIDDM) patients with nephropathy. Clin Genet 48: 288-292

32. Taskinen M-R, Kahri J, Koivisto V, Shepherd J, Packard CJ (1992) Metabolism of HDL apolipoprotein A-I and A-II in Type 1 (insulin-dependent) diabetes mellitus. Diabetologia 35: $347-356$

33. Eisenberg S (1985) Preferential enrichment of large-sized very low density lipoprotein populations with transferred cholesteryl esters. J Lipid Res 26: 487-494

34. Tall A, Sammett D, Granot E (1986) Mechanisms of enhanced cholesteryl ester transfer from high density lipopro- 
teins to apolipoprotein B-containing lipoproteins during alimentary lipemia. J Clin Invest 77: 1163-1172

35. Mann CJ, Yen FT, Grant AM, Bihain BE (1991) Mechanism of plasma cholesteryl ester transfer in hypertriglyceridemia. J Clin Invest 88: 2059-2066

36. Tamsma JT, Beverdam FH, Leuven JA, Lemkes HH (1995) Decreased interstitial apolipoprotein A-I levels in IDDM patients with diabetic nephropathy. Diabetes 44: 501-505

37. Clodi M, Oberbauer R, Bodlaj G, Hofmann J, Maurer G, Kostner K (1999) Urinary excretion of apolipoprotein (a) fragments in type I diabetes mellitus patients. Metabolism 48: $369-372$
38. Coonrod BA, Ellis D, Becker DJ et al. (1993) Predictors of microalbuminuria in individuals with IDDM. Pittsburgh Epidemiology of Diabetes Complications Study. Diabetes Care 16: 1376-1383

39. Horita K, Eto M, Makino I (1994) Apolipoprotein E2, renal failure and lipid abnormalities in non-insulin dependent diabetes mellitus. Atherosclerosis 107: 203-211

40. Boize R, Benhamou PY, Corticelli P, Valenti K, Bosson JL, Halimi S (1998) Apo E polymorphism and albuminuria in diabetes mellitus: a role for LDL in the development of nephropathy in NIDDM? Nephrol Dial Transplant 13: $72-75$ 